\title{
LA-UR-17-25563
}

Approved for public release; distribution is unlimited.

Title: $\quad$ Exploring the Use of Activity Patterns for Smart Monitoring of Nuclear Facilities

Author(s): $\quad$ Miller, Karen Ann

Intended for: Visitor Presentation

Issued: 
Disclaimer:

Los Alamos National Laboratory, an affirmative action/equal opportunity employer, is operated by the Los Alamos National Security, LLC for the National Nuclear Security Administration of the U.S. Department of Energy under contract DE-AC52-06NA25396. By approving this article, the publisher recognizes that the U.S. Government retains nonexclusive, royalty-free license to publish or reproduce the published form of this contribution, or to allow others to do so, for U.S. Government purposes. Los Alamos National Laboratory requests that the publisher identify this article as work performed under the auspices of the U.S. Department of Energy. Los Alamos National Laboratory strongly supports academic freedom and a researcher's right to publish; as an institution, however, the Laboratory does not endorse the viewpoint of a publication or guarantee its technical correctness. 


\section{Exploring the Use of Activity Patterns for Smart Monitoring of Nuclear Facilities}

July 2017

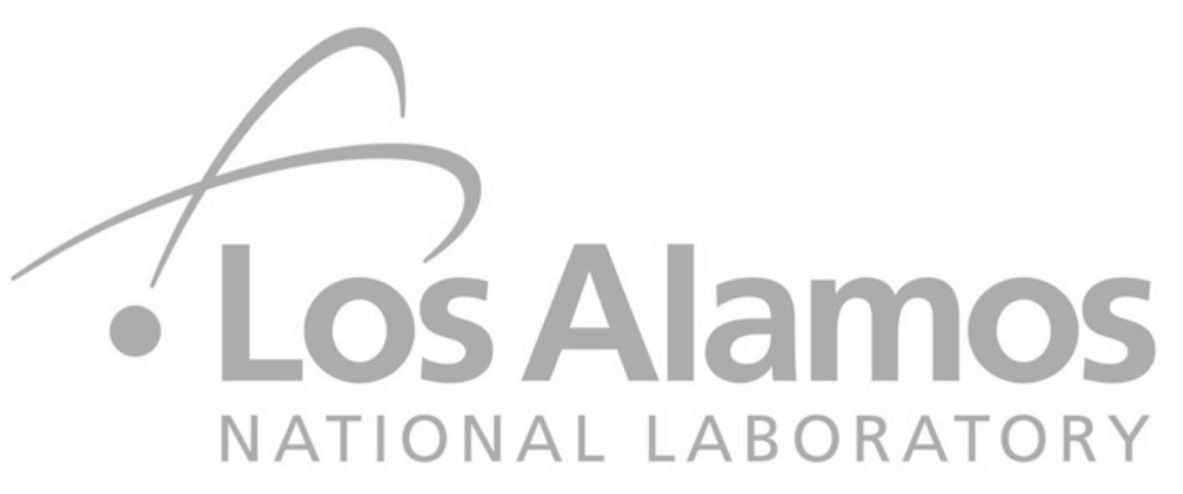

Dr. Karen Miller

Safeguards Science \& Technology 


\section{Bottom Line}

The world is at an inflection point where our ability to collect data now far outpaces our ability to make use of it

LANL has a number of efforts to help us pull more meaningful insights out of our data and target resources to where they will be most impactful

We are exploring an approach to recognizing activity patterns across disparate data streams for a more holistic view of nuclear facility monitoring 


\section{Positioning for the Future}

If you went to bed last night as an industrial company, you're going to wake up today as a software and analytics company.

- Jeff Immelt, CEO General Electric

LANE has a wide range of nuclear facilities used for experiments, training, manufacturing, and waste handling

Can we use a combination of persistent sensing and other data we're already collecting to improve safety, security, safeguards, and operational efficiency? 


\section{Why now?}

A perfect storm of technology advances in commercial industry has led to a flywheel effect that is increasing innovation in this area at an unprecedented rate
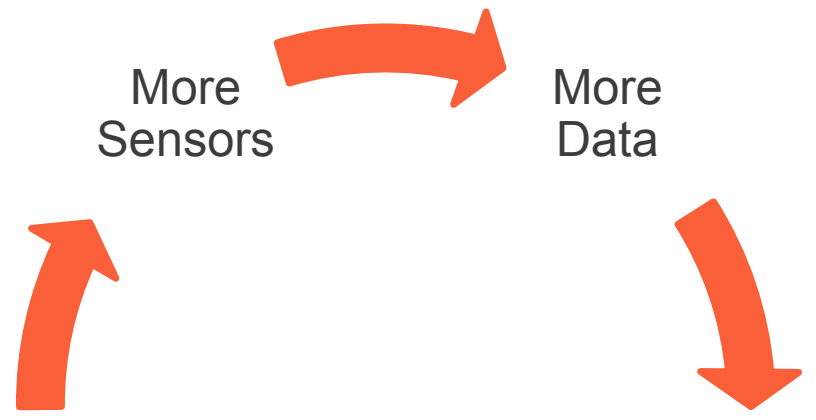

Better

Algorithms

More Computing

Infrastructure

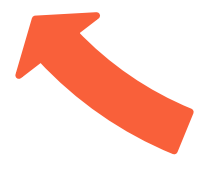

More

Processing 


\section{Characterizing Activity Patterns}

Characterizing activity patterns provides us a baseline to define "normal" so we can identify early indicators of events and cue on things that are "abnormal"

Persistent sensing has opened the door for us to move beyond static signatures to characterizing dynamic activities in aggregate over time and space

Diversity in sensing modalities and other data sources can help bring missing context to a situation and improve detection probability and reduce false alarm rate

The challenge lies in automating the analysis to allow us to do this at scale, in real time, and with some measure of uncertainty 


\section{Applications in Other Domains}

Activity Recognition Based on Inertial Sensors for

Ambient Assisted Living

Why Fitbit's Auto-Activity Tracking is a Big Deal

Complex Activity Recognition using Granger Constrained DBN (GCDBN) in Sports and Surveillance Video

User activity recognition for energy saving in smart homes

Next-Gen Cybersecurity Is All About Behavior Recognition
Establishing Fraud Detection Patterns Based on Signatures

Recognizing Wide-Area and Process-Type Activities 


\section{Activity Patterns in Nuclear Facility Monitoring}

- Challenge is obtaining ground-truthed data sets for testing approaches

- LANL has control over and knowledge of activities at our facilities

- We are able to use them as testbeds for developing validated methodologies

- Approach: crawl - walk - run

- Start with smaller-scale studies and incorporate insights and lessons learned into larger campaigns

- Proof-of-concept measurement campaigns

- Dual-Axis Radiographic Hydrodynamic Test Facility (DARHT)

- Plutonium Processing Facility (PF-4)
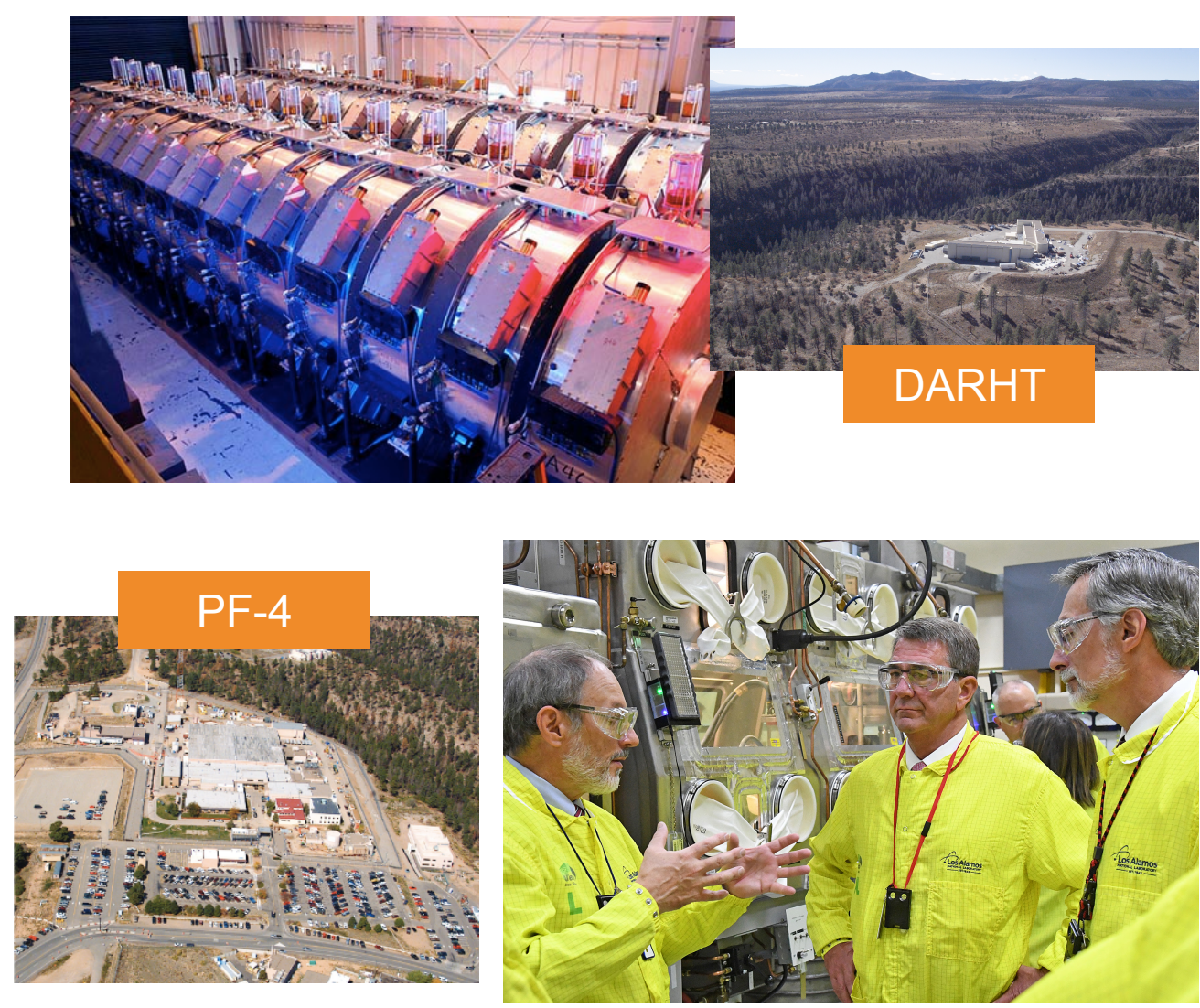


\section{Measurement Campaigns}

Included multidisciplinary teams of experts in facility operations, sensor technologies, signature characterization, statistics, and data science

\section{DARHT}

- Six months of data collection

- Data streams

- Seismic sensor

- Acoustic sensor

- Radiofrequency sensor

- Surveillance video

- Internal network traffic

\section{PF-4}

- Two weeks of data collection

- Data streams

- Neutron detector

- Radiofrequency sensor

- Seismic sensor

- Internal network traffic

- Vehicle logs

- Criticality database 


\section{Preliminary Results}

\section{The whole is greater than the sum of its parts}

How to efficiently combine data streams to come to a credible assessment?

Successful demonstration of disparate data integration, feature extraction, and predictive modeling of activities of interest at DARHT and PF-4

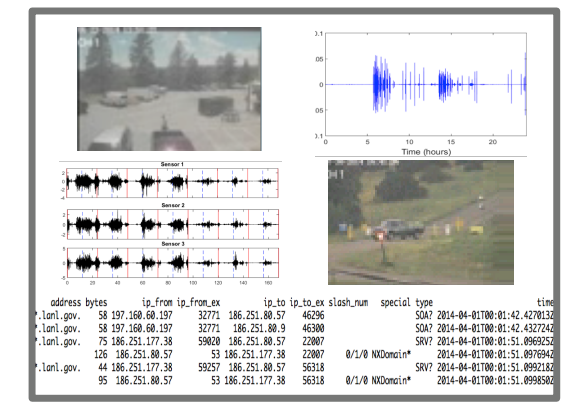

Disparate Data Streams

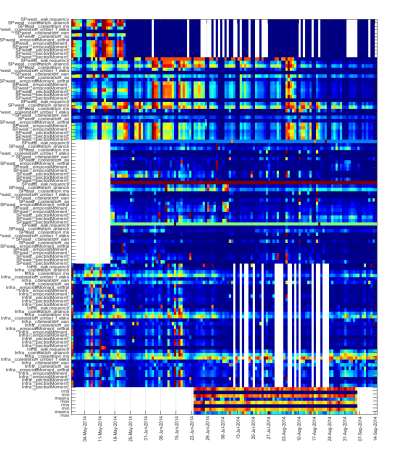

Feature Extraction

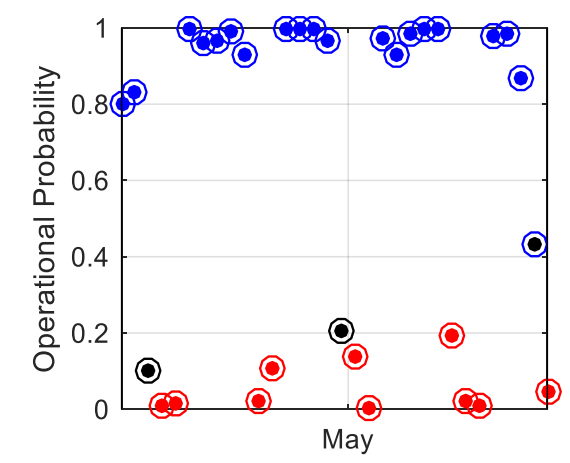

Predictive Modeling
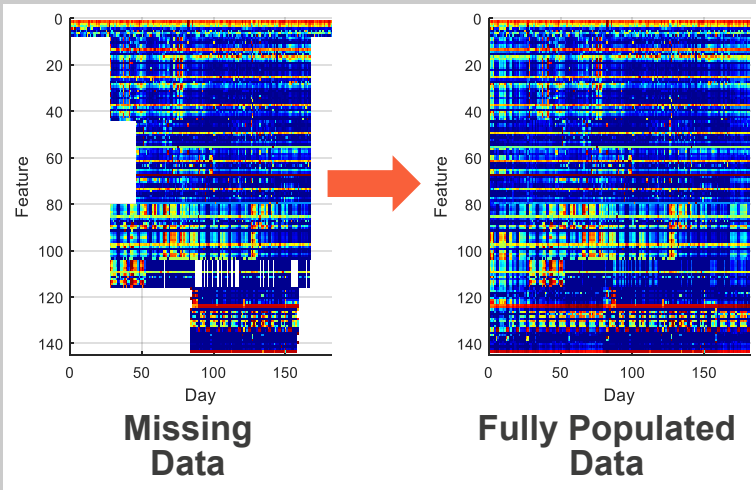

How to deal with missing or incomplete data?

Exploration of statistical methods that utilize the underlying correlation structure and track uncertainty over time 


\section{Potential Next Steps}

\section{- Follow-on work}

- Expand sensor network to the TA-66 schoolhouse to characterize training activities

- Refine machine learning techniques for activity characterization across disparate data streams

- Develop methodologies for dynamic assessment of data quality and uncertainty quantification

- Insertion of adversarial scenarios to evaluate robustness to negative examples

- Test generalizability of approaches through application to diverse facilities

- Potential to install persistent sensors in facilities as they undergo renovations
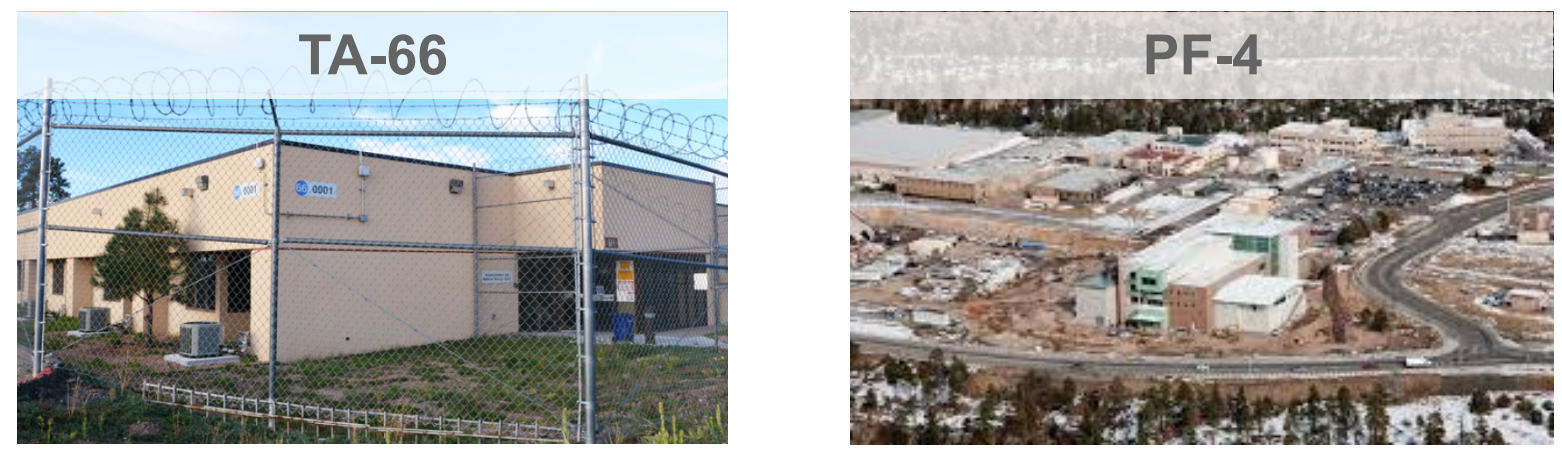

\section{Other Facilities}

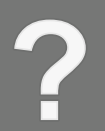




\section{Expanding the lens beyond facility monitoring}

Understanding geospatial-temporal trends in Lab-wide safety data to increase situational awareness and enable anticipatory analytics

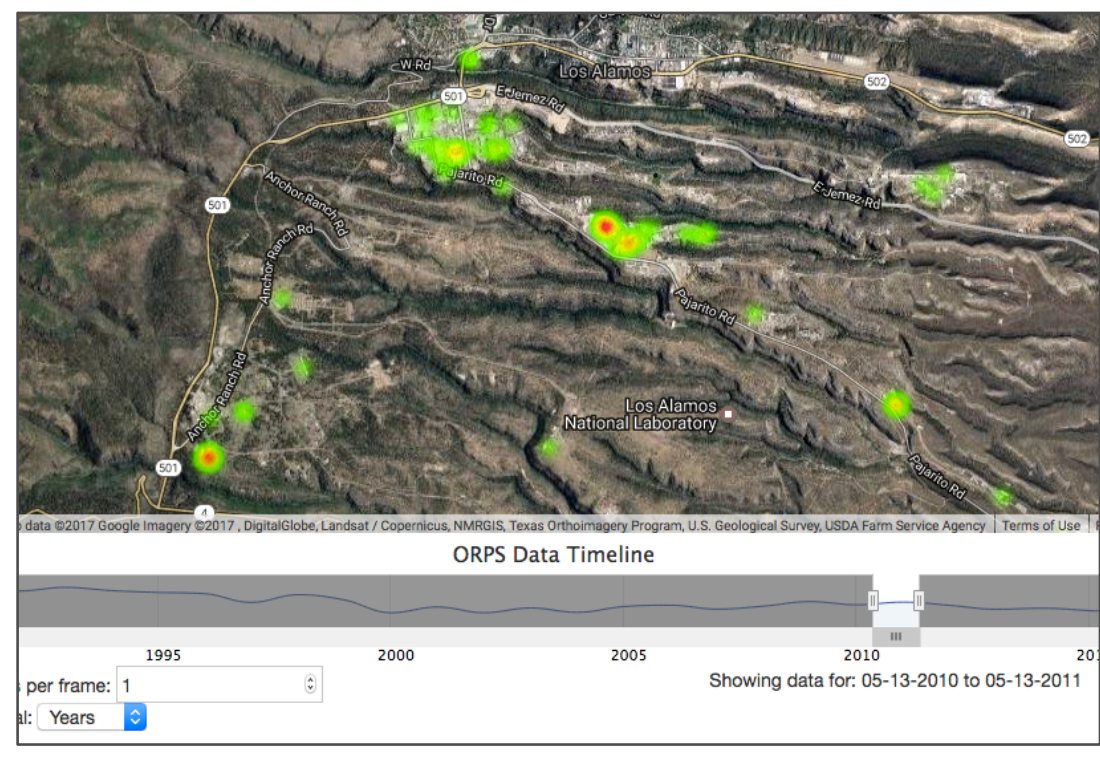

Interactive heatmap of incident locations with time window slider

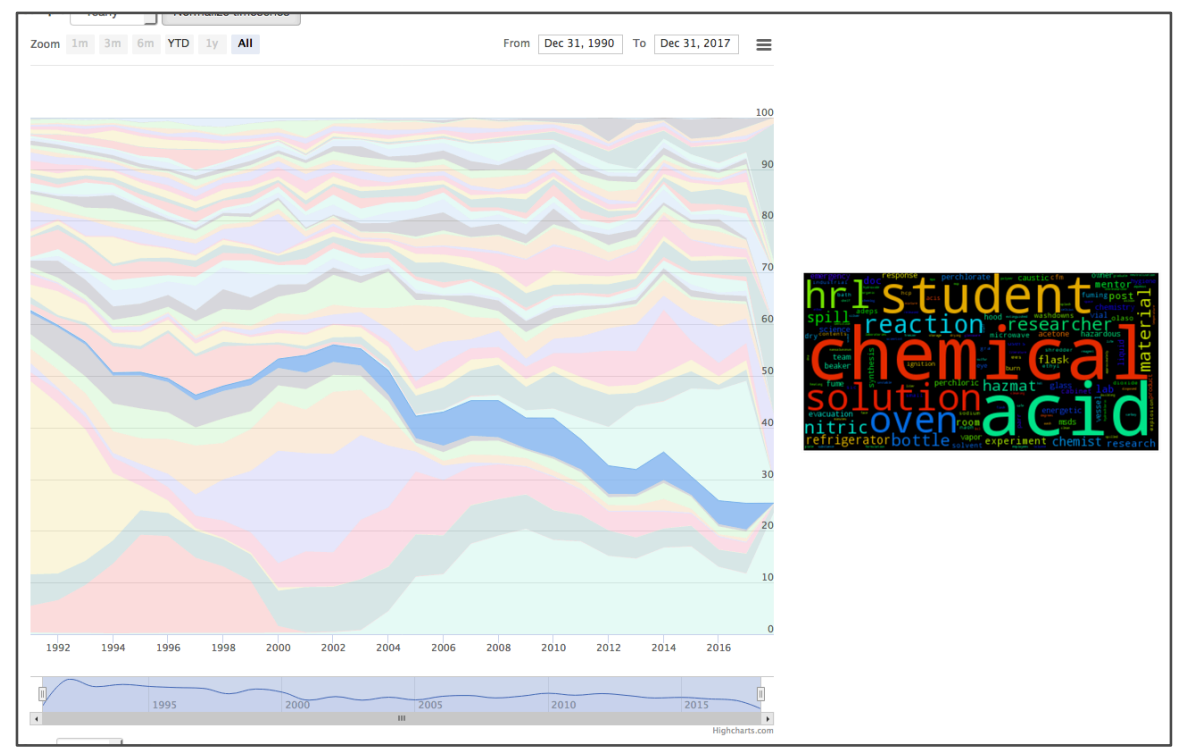

Text analytics used to automatically cluster free-text reports into categories and visualized over 25 years 


\section{Expanding the lens... even farther}

- The number of small satellites in orbit is expected to surpass 1,500 this year

- Global daily coverage $\rightarrow$ how to make use of increased temporal information?

- Exploring approaches to automated change detection using public synthetic aperture radar (SAR) data

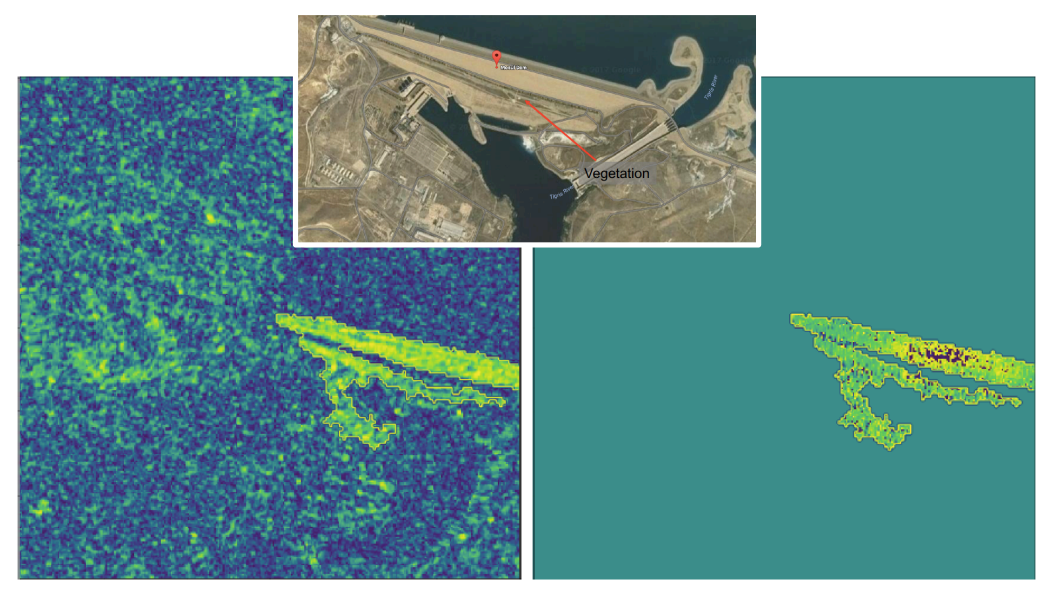

Subsidence detection applied to the failing Mosul Dam using open-source machine learning tools

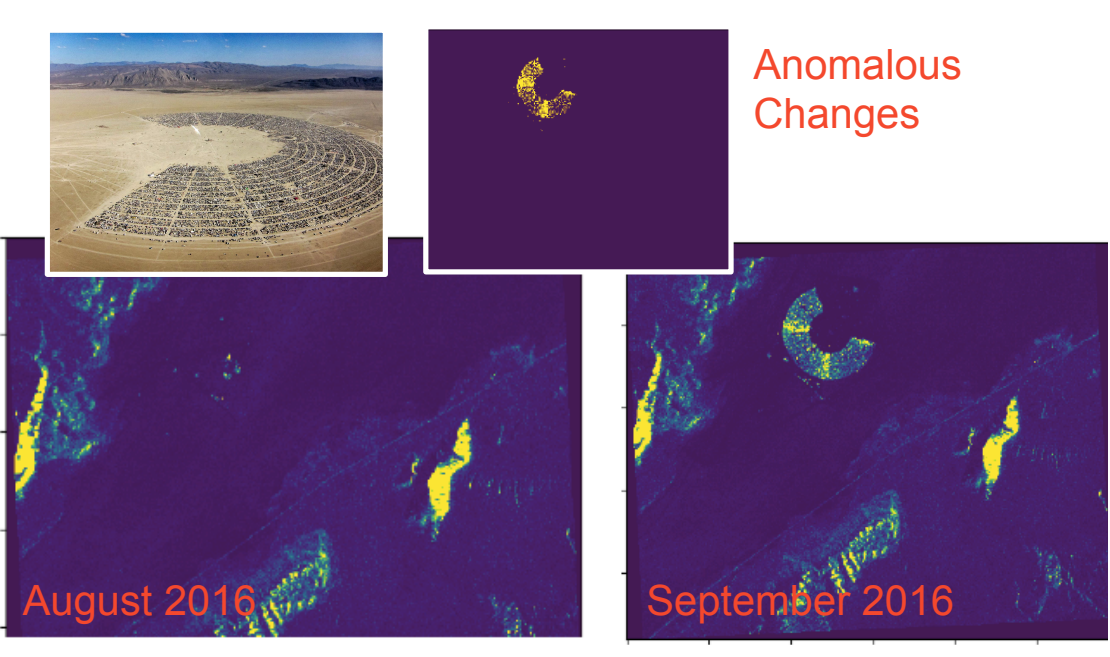

Anomalous change detection algorithm applied to before and during SAR images of a week-long festival in Nevada 


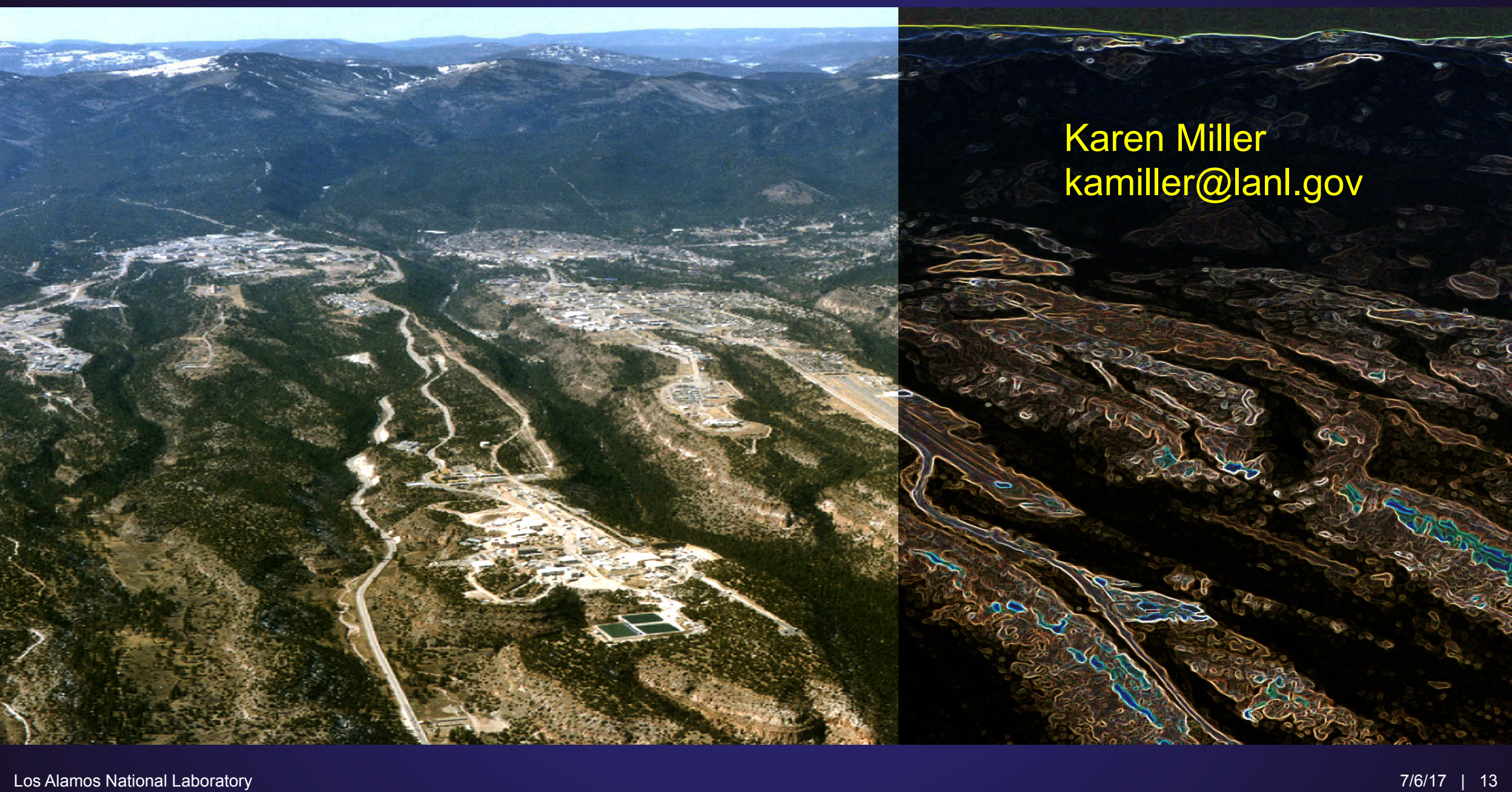

